\title{
Contact-Induced Change in an Oceanic Language: The Paluai - Tok Pisin Case
}

\author{
Dineke Schokkin \\ The Australian National University \\ dineke.schokkin@anu.edu.au
}

\begin{abstract}
Many studies have focused on substrate influence on the creole languages of Melanesia - Tok Pisin, Solomons Pijin and Bislama. The same cannot be said with regard to influence in the opposite direction: contact-induced change occurring in local vernaculars due to pressure from the creole. This paper presents a case study of several instances of structural borrowing and semantic category change in Paluai, an Oceanic language spoken in Papua New Guinea. It is shown that a number of functional elements originating from Tok Pisin are now firmly embedded in Paluai grammar: two verbs, gat and inap, and a conjunction, taim. Moreover, semantic categories are undergoing change and possibly attrition due to many-to-one correspondences. This suggests that it is important to view language contact situations as dynamic and involving two-way processes of change.
\end{abstract}

\section{Keywords}

structural borrowing - creole languages - language contact

\section{$1 \quad$ Introduction}

This paper discusses a number of phenomena in Paluai (affiliation: Austronesian, Oceanic, Admiralties), spoken on Baluan, a small island in Manus Province, Papua New Guinea (PNG), which may have arisen due to contact with and transfer from Tok Pisin (TP), a creole spoken as lingua franca in the area. Paluai is more commonly known and referred to as Baluan-Pam (Iso 639-3: blq), but is preferably referred to by the speech community as Paluai. This practice will be followed through this paper. 
PNG is one of the linguistically most diverse countries in the world, both in terms of number of languages relative to population size and in terms of unrelated phyla (cf. Nettle (1999)). Many of its languages have a small to very small number of speakers and can be classified as endangered, due to lack of intergenerational transmission and the high pressure of more dominant languages, in particular English and TP.

In the paper, the focus will lie on which elements from тP are encountered in Paluai, what their functions are and possible explanations as to why they were borrowed. Substrate influence from Oceanic languages on тP and the closely related creole languages Solomons Pijin and Bislama has been well documented (see Crowley, 1990; Keesing, 1988, 1991; Meyerhoff, 2000, 2001, 2009; Romaine, 1992a, 1995; Sankoff, 1994; Siegel, 1998 and Smith, 2002, inter alia). However, change in the opposite direction, i.e. influence from TP on local vernaculars, seems to have been largely overlooked in the literature on language contact (but see Ross, 1985, and Jenkins, 2005), although individual reference grammars may include sections dedicated to this.

The current paper will discuss several cases of structural borrowing (borrowing of grammatical forms) from TP into Paluai. There will also be a discussion of semantic category change and attrition due to a many-toone correspondence of semantic categories in Paluai and тP, respectively. Data on which the analyses are based were gathered by the author during two extensive field trips on Baluan Island in 2010-11 and 2012. The data were primarily intended for a language documentation project as part of a $\mathrm{PhD}$, and therefore the current paper does not include any quantitative analyses. It will, however, make some inroad in determining what the situation on the ground looks like, and hopefully pave the way for future studies, in this particular speech community or other ones, that will supplement the perhaps somewhat impressionistic discussion in this paper with more robust findings.

In the final part of the paper, some broader questions relating to contactinduced change and language shift will be touched upon. What does the increasing pressure of contact languages on local vernaculars mean for their future? Could creole languages be 'special cases' when it comes to borrowing grammatical items? Does contact-induced change actually decrease or increase language variation?

On Baluan Island, two languages are spoken: Paluai (of which a variety is spoken on nearly Pam Island) by the majority of inhabitants, and Titan by 
a minority living in one village on the northeast coast of the island. ${ }^{1}$ The Titan speakers are fairly recent immigrants, and their presence does not seem to have had noticeable influence on Paluai, except for a few loanwords. Every member of the Paluai-speaking community acquires the language from birth as their native language. In addition, people acquire TP, an English lexifier creole that is an official language of PNG, often from a very early age. Parents, on the whole, speak Paluai to their children, but with frequent code switching into TP. Mostly, however, children seem to pick up TP from their slightly older peers. Informal observation of groups of playing children reveals that they use mostly TP to communicate; many children probably receive a significant proportion of their input in this kind of communicative situation rather than from the parental generation. The importance of slightly older children and adolescents - as opposed to infants - in the emergence of new varieties has been noted by a range of authors; see e.g. Kerswill (1996), Kerswill and Williams (2005), Aitchison (2003) and Ross (2013). As Kerswill (1996: 198) notes:

Adolescents are clearly significant bearers of change; their networks allow them to have wider contacts than younger children, and their desire for a distinct social identity means that they are willing to modify their speech. At the same time, during this period (at age 16 at the very latest) they no longer have the ability to acquire lexically complex rules, new oppositions, or new intonational systems.

English, although gaining ground as the language of mass media, government and education, is not commonly acquired in a naturalistic setting by people on the island, and people's first exposure to English is usually when they enter primary school around the age of eight. People of the older generations (about age forty and upwards) often show at least a passive command of one or more of the surrounding languages, such as Titan and Lou, due to long-standing contacts between various neighbouring speech communities. These forms of passive multilingualism are on their way out, since TP is increasingly used as a lingua franca between language groups.

TP is dominant over Paluai in terms of its absolute number of speakers and the domains in which it is used. It is the language of the church, and one of the official languages of PNG, which gives it a certain prestige. On the other hand, English is seen as the language that is needed to get ahead in the world, the one that one needs to master in order to get a proper education and access

1 Titan is spoken in at least seven different locations in the coastal regions of Manus Island and several of its surrounding islands. 
to white-collar jobs. In this respect TP is clearly seen as inferior to English, but also in a sense to the local vernaculars. It is commonly referred to as 'not a proper language', 'vague' and 'broken'. Still, it may have covert prestige as the main language of urbanised young people, social media and popular culture and music; it thus may have a strong attraction for pre-adolescent and adolescent speakers who can play, as noted above, a significant role in language change. On the whole, people have a very pragmatic attitude about the use of TP and see it mainly as a way to facilitate communication between members of different language groups. Nowadays, however, the language is frequently used in intragroup communication as well, leading to extensive code switching and borrowing.

When we look at language vitality of Paluai, there are several important factors. Even though the language scores well on intergenerational transmission and all speech community members still speak the language, it is under severe pressure from TP and, to a lesser extent, English. Although diglossia and multilingualism do not necessarily have to lead to language loss, with TP there maybe an additional aggravating factor: it has an Oceanic substratum, most of which can be traced back to Kuanua (Tolai) (Mosel, 1980). Therefore, it shows many structural similarities to the vernaculars of Manus Province. Because the languages are structurally so similar, there is a high likelihood of transfer and calquing, which in turn may lead to further convergence of the languages in question. In addition, children start using TP so early in life that the situation can most adequately be described as bilingual acquisition of Paluai and TP, which again is a circumstance with a high likelihood, in the long run, of convergence of the languages involved.

\section{3}

\section{Borrowing}

\subsection{Lexical Borrowing}

When taking into account borrowing phenomena, it is important to make a distinction between lexical and structural borrowing. Lexical borrowing entails the insertion of lexical items from one language into another. This phenomenon is very common in all languages of the world. It can be done for various reasons. For instance, when a speech community does not have a word for a specific object or concept, because this is newly introduced, it will usually adopt a term from a dominant foreign language for it. This has happened in Paluai with many items and concepts that were introduced during the colonial period and new technologies that are still being introduced (e.g. pencil, car, school, mobile). Previously, these lexical items usually entered the language 
'through' TP: since TP is an English lexifier creole, it adopted many lexical items from English. Nowadays, more and more items are adopted into Paluai directly from English, since this has become the predominant language of education and mass media. A feature of loanwords is that they are adapted to the recipient language's phonotactics and morphology.

Lexical borrowing for 'foreign' objects and concepts, however, can be regarded as rather trivial. It is a pervasive phenomenon in any language, and as long as the language is strong in other respects, it usually does not lead to language obsolescence. Sometimes, however, lexical borrowing also takes place even though the recipient language has a term for the concept. This is also the case in Paluai: very frequent are, for instance, TP wasim 'to wash', hangamap 'to hang, be hanging' and stori '(to tell) a story'. That they are regular lexical borrowings, rather than instances of code switching, is shown by the fact that they are subjected to Paluai phonotactics and morphosyntax. Wasim, for instance, is encountered with the instrumental applicative suffix -ek: wasimek 'to wash with'.

It is an intriguing question why a loanword would be used in cases where a vernacular term is readily available. Below, where the phenomenon of semantic category change is discussed, we will come back to this question, and as it turns out there may be a principled reason behind a number of these lexical borrowings.

\subsection{Structural Borrowing}

Structural borrowing entails the adoption of grammatical or functional items from a donor language into a recipient language. This is much rarer than lexical borrowing, and it usually only occurs when two languages are in intensive contact for an extended period of time, i.e. when a speech community is genuinely bilingual in both languages and uses them together, which means that there is a large amount of code switching, and children acquire both languages at an early age. Structural borrowing is thought to be more likely to happen if the two languages are typologically similar: Thomason and Kaufman (1988: 54) hypothesize that 'in cases of light to moderate structural interference, the transferred languages are more likely to be those that fit well typologically with corresponding features in the recipient language.' Since $\mathrm{TP}$ has an Oceanic substrate, it is structurally very similar to Paluai and other Admiralties languages, and indeed we do find a number of examples of structural borrowing. Sometimes, but not always, structural borrowings fulfil functions for which there is an omission in the recipient language, i.e. where there is no grammaticalized form or construction in this language. In what follows, three functional items 
that were borrowed from TP into Paluai will be discussed. These consist of two verbs, the possessive/existential verb gat 'have' and the modal verb inap 'be able', and the temporal subordinate clause marker taim 'when'.

\subsubsection{The Verb Gat}

Paluai does not have a verb expressing predicative possession, comparable to English 'have'. This hiatus in the grammar has been filled by the TP form gat (which is based on English get/got). Normally, possession is indicated within the NP, by means of a 'pertensive' suffix expressing person and number of the possessor either directly on the noun (in the case of most body parts, kinship terms and other 'inalienable' entities) or on a particle directly following the possessee noun (in other cases).

Not only is gat used for possessive predicates, it also functions to mark existential predicates, in particular negated ones. This existential function of gat is encountered in TP too, and thus not an innovation in Paluai. Below, examples typical of the possessive and existential uses of gat in TP are given.
(1) mi gat wanpela sista 1sg have one/a sister 'I have one/a sister.'

$\begin{array}{lll}i & \text { gat } & \text { suka long stua? } \\ \text { PRED have sugar LOC } & \text { store }\end{array}$

'Is there sugar in the store?'

In Paluai possessive constructions, gat is used as in the example below:
(3) ngagat kokon
nga $=$ gat kokoni
1sg=have money
'I have money'

2 The тр form $i$, together with its Solomons Pijin and Bislama counterparts, is labeled a 'predicate marker' by most authors (see e.g. Crowley (2000) for a discussion of various analytical options). It is possible that this TP form has been reanalysed as the Paluai 3 sg personal pronoun $y i$ when verbs are borrowed from TP. The fact that gat and inap occur with a 'dummy' 3 sg subject, a feature unique to them (i.e., not attested with any 'native' verbs), also seems to suggest this. 
A Paluai affirmative existential clause is formed using the verb to $(k)$ 'to be, to stay, to exist'. ${ }^{3}$ An example is given below, consisting of a presentational sentence from the introductory part of a narrative.

(4) mui a ngoyai, urok

mui a ngoyai $\mathrm{u}=$ tok

dog and possum $3 \mathrm{du}=\mathrm{be}$

'(Once upon a time,) there were a dog and a possum.' (LL010711_0oo6)

However, this construction seems to be on its way out and replaced by a construction with TP gat with a dummy 3 sg subject $y i-$, as in example (5) below. Here, gat is in fact superfluous; the sentence would also be grammatical if it would be left out. Note that la does not indicate motion, but rather distance from the deictic centre. Also interesting is the observation that Paluai does not make use of dummy subject constructions except in this case and in the case of inap, another loan from TP, discussed below. Thus, not only have new function words entered the language, a new syntactic construction seemingly has entered the language with them.

(5) igat nik laro net pulek

yi=gat nik la-to net pulek

3 sg=have fish go.to-be sea too

'There is fish in the sea too.' (o52b_0308)

Existential clauses with tok, such as example (4), do not seem to be negated. ${ }^{4}$ Rather, to express predicatively that something is not there, speakers seem to prefer the abovementioned clause with gat, with a ssg dummy subject, which is then negated. Two examples are given below.

(6) imagatyon pwên

yi=ma=gat [yanu $]$ pwên

$3 \mathrm{sg}=\mathrm{NEG}_{1}=$ have $\quad$ water $\quad \mathrm{NEG}_{2}$

'There was no water.' (NS220511_1_oo32)

3 The full verb form is tok, whereas to is used when there is a constituent (such as a locative) in addition to the verb.

4 Negation in Paluai is discontinuous, with the element $\mathrm{ma}$ - placed between the subject bound pronoun and the verb, and the particle pwên placed directly following the material over which the negation has scope. 
(7) imagat somyamat te iro pwalinganip pwên

\begin{tabular}{|c|c|c|c|c|}
\hline $\begin{array}{l}y i=m a=g a t \\
3 s g=N E G_{1}=\text { have }\end{array}$ & $\begin{array}{l}\text { [som } \\
\text { one.ANIM }\end{array}$ & $\begin{array}{l}\text { yamat } \\
\text { person }\end{array}$ & $\begin{array}{l}\text { [te } \\
\text { REL }\end{array}$ & $\begin{array}{l}y i=\text { to } \\
3 \text { sg=be }\end{array}$ \\
\hline pwalinga-n-ip]] & pwên & & & \\
\hline with-PERT-3pl & $\mathrm{NEG}_{2}$ & & & \\
\hline
\end{tabular}

There may be a pragmatic reason why gat is gaining ground in existential constructions, which has to do with preferred information structure. The main function of existential clauses is to introduce discourse participants, as brandnew information. This is the case in all the examples above. In example (4), the 'traditional' Paluai construction, the introduced discourse participants mui 'dog' and ngoyai 'possum' occupy the sentence-initial position, as S arguments of the verb tok. Lambrecht (1994) discusses the ways in which brand-new information can be introduced. Sentence-initial position is normally associated with the sentence topic (readily accessible information, identifiable for both speaker and hearer), and thus does not lend itself easily for expression of brand-new information, which is unidentifiable for the hearer. Therefore, often a construction will be used in which another element occupies the sentenceinitial slot. For instance in Dutch, an indefinite NP, by default containing information not identifiable to the hearer, sits uneasily in sentence-initial position. ?Een paard staat in de gang 'A horse stands in the corridor' can only have generic interpretation, referring to the category of horses as a whole, but an inverted construction with the dummy element $e r$ is much preferred and correct interpretation is much easier: Er staat een paard in de gang 'There is a horse in the corridor'.

Thus, the 'original' Paluai structure in (4) is less fortunate in terms of information structure: the brand-new information occupies sentence-initial position. When, instead, this kind of sentence is expressed with an igat construction, the brand-new element is moved from the problematic sentenceinitial position to postverbal position, as $\mathrm{O}$ argument of the verb $g a t$, which has a third person singular dummy subject $y i$ - This is the case in examples (5), (6) and (7). In this way, the construction is possibly more in line with general pragmatic preferences of having the brand-new information in postverbal rather than sentence-initial position. It is possible that the advance of igat constructions started off in sentences with negative polarity, for which it now seems to be the only permissible way to express them, and that from there it spread to environments with positive polarity. 
3.2.2 The Verb Inap

The lexical material for the verb inap comes from English 'enough', but its semantic and grammatical properties are thoroughly substrate. It can have the meaning '(be) sufficient', but also has a modal meaning, in which case it either has an interpretation of ability or of possibility. Below, two examples of the use of inap in TP are given.

$\begin{array}{lllll}\text { (8) } & \text { trausis bai inap long en } \\ \text { trousers } & \text { FUT sufficient } & \text { LOC } & 3 \text { sg } \\ \text { 'The trousers will fit him.' } & & \end{array}$
(9) mi inap harim tokples
1sg able understand vernacular
'I can understand the local language.'

In both cases it takes a complement clause (CC), but this is looking slightly different for each interpretation. In Paluai, cc's are usually marked by either te, in case of a CC with transitive object $(\mathrm{O})$ function, or a-te for a CC with peripheral argument (Oblique) function; this formal distinction generally corresponds to a semantic distinction between Fact and Activity CC's respectively (see Dixon (2012) for discussion of semantic types of CC's, and Schokkin (2014) for a more detailed discussion of complementation). There is a third type of cc, formally marked with irrealis, which corresponds to Potential semantics.

When inap is referring to ability, it is usually marked with a Potential cc, as in examples (10) and (11) below. In some cases, it is attested with a complement showing a nominalisation, as in (12). Note that although examples (10) and (11) are negative and (12) is interrogative, inap also occurs in declarative clauses.

(10) ngamainap kope pe pwên

$$
\begin{array}{llll}
\text { nga=ma=inap } & \text { [ko-pe } & \text { pe=Ø } & \text { pwên }] \\
\text { 1sg=NEG }=\text { able } & \text { IRR.1sg-PFV } & \text { do=3sg.zERo } & \text { NEG }_{2} \\
\text { 'I am not able to do it.' (LLO30611_oo33) } &
\end{array}
$$

(11) ipmainap kape roui lai pwên

$$
\begin{aligned}
& \text { ip=ma=inap [ka-pe tou=i la a-yi pwên] } \\
& 3 \mathrm{pl}=\mathrm{NEG}_{1}=\mathrm{able} \quad \text { IRR.NS}-\mathrm{PFV} \text { put }=3 \text { sg go.to at-3sg } \mathrm{NEG}_{2} \\
& \text { 'They were not able to put him into it.' (Game1_021012_049o) }
\end{aligned}
$$


(12) ngainap ai songanum le papwên?

$\begin{array}{llllll}\text { nga=inap } & \text { a-yi } & \text { [song-an } & \text { wumwa] } & \text { le } & \text { papwên } \\ 1 \text { sg=able } & \text { at-3sg } & \text { run.away-Nom } & \text { house } & \text { or } & \text { not.yet }\end{array}$

'Am I able to get married or not yet?' (KW290611_0031)

When inap is referring to possibility, there is a 3sg dummy subject in the main clause, and the complement clause is marked with $(a-) t e$ in addition to being marked for irrealis. inap can have a CC with either $\mathrm{O}$ or Oblique syntactic function, i.e. either marked by te or marked by $a$-te. However, the distinction between Fact and Activity is a bit tentative in these cases, since their foremost characteristic is that they can be regarded Potential cc's, and are correspondingly marked for irrealis. Below, two examples of inap are given with an O CC, followed by two examples of inap with an Oblique cc.

(13) kaba iinap te no wo ono apwut, ya woapwut kapwa yi=inap [te no wo wo=no apwut] if $\quad 3$ sg $=$ able comP only you 2 sg $=\mathrm{IPFV}$ clear.bush

ya wo=apwut

then 2 sg=clear.bush

'If it is possible that you clear the bush (for a garden) by yourself, alright then you will clear the bush.' (KM 190211_0007)

(14) imainap te muyan kipe palak liliu pwên

$\begin{array}{llllll}\mathrm{yi}=\mathrm{ma}=\mathrm{inap} & {[\mathrm{te}} & {[\text { muya-n }]} & \text { ki-pe } & \text { palak } & \text { liliu }] \\ 3 \mathrm{Sg}=\mathrm{NEG}_{1}=\text { able } & \text { COMP } & \text { skin-PERT } & \text { IRR.3sg-PFV } & \text { bad } & \text { again }\end{array}$

pwên

$\mathrm{NEG}_{2}$

'It is not possible that his skin will go bad again.'(NP260511_oo24)

(15) woning te iinap are yep kino rer ai

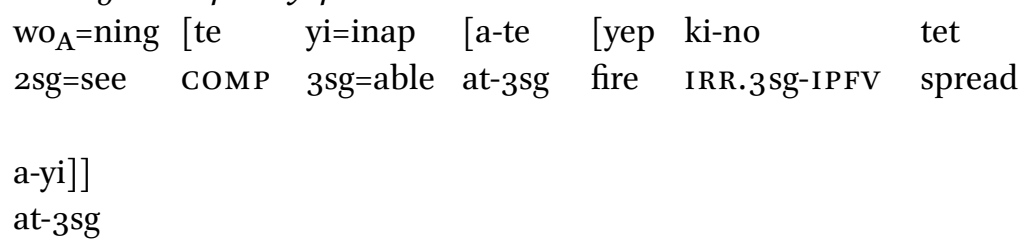

'You see that it is possible for fire to spread through it.'(KM190211_0017) 
(16) imainap are ip silal kape wau tarak toui a kape lêpi pwên

$\begin{array}{llllll}\text { yi=ma=inap } & {[\text { a-te }} & {[\text { ip }} & \text { silal }] & \text { ka-pe } & \text { wau } \\ 3 \text { sg=NEG } 1=\text { able } & \text { at-сOMP } & \text { 3pl } & \text { spirit } & \text { IRR.NS-PFV } & \text { move }\end{array}$

$\begin{array}{lllll}\begin{array}{l}\text { tarak tou }=\mathrm{i} \\ \text { climb give=3sg }\end{array} & \text { a } & \text { ka-pe } & \text { lêp=i] } & \text { pwên } \\ \text { IRR.NS-PFV } & \text { take=3sg } & \text { NEG }_{2}\end{array}$

'It was not possible that the spirits would follow him into the tree and get him.' (LM190611_0041)

Thus, there seems to be a clear formal difference between inap clauses referring to ability and those referring to possibility, with the first just being marked by irrealis, and showing coreference between the S/A argument of the main and the dependent clause, and the second being marked by both $(a-) t e$ and irrealis. It is unclear why the latter can take both $a$-te and te as a complementizer. It is possible that inap CC's have variable marking because they always take Potential Cc's, which are already marked by irrealis, and which are not marked by $(a-)$ te for the other Potential complement clausetaking verb in Paluai, $p w a$ 'want to, be going to'. The fact that there is variation (no marking, marking with te and marking with $a-t e$ ) indicates that inap may not yet be fully integrated into Paluai syntax and therefore exhibits unpredictable behaviour.

It can be concluded, however, that inap functions as a deontic modal operator (Timberlake, 2007: 329) indicating ability and/or possibility. The authority can either be the participant designated to carry out the action, who does not have the (physical) ability to do it, or the participant may not be able to do it due to external circumstances. inap as a modal operator does not fill a hiatus in Paluai grammar; the 'indigenous' form $s a$ can fulfil the same function, as example (17) below shows. Non-negated $s a$, however, has apprehensive overtones, which are absent from inap. The first, negated, use of $s a$ indicates an impossibility, whereas the second, non-negated, use refers to a possible outcome which would be undesirable. This may be a reason why inap is gaining ground as a modal operator: the apprehensive overtones that $s a$ carries seem to be absent from inap.

(17) ngasa pul la rai pwên, te isa yeki

nga=sa pul la ta-i pwên te yi=sa yek=i 1 sg=MOD speak go.to POss-3sg NEG SUB 3sg=MOD hit=3sg 'I cannot tell him, lest he beat her/for he may beat her.' (WL020711_oo56) 


\subsubsection{The Subordinator Taim}

The form taim ('when', lit. 'time') indicates co-occurrence or contiguity of events without a strong cause-effect relation (although overtones of this can be present).

Below, an example is given of the use of taim in TP.
taim kopi $i$ kol liklik yu dringim when coffee PRED cold little 2sg drink.TR
'When the coffee has cooled down a little, you drink it.'

taim marks a temporal subordinate clause which precedes the main clause, and the main clause is often marked by $y a$ 'then'. Below, two examples are given.

(19) taim te imwat teo, a wope antek

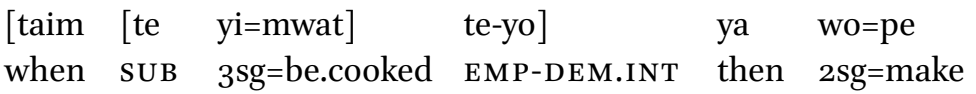

antek=Ø

put.away $=3$ sg.zERo

'When it is cooked ready, you will put (it) away.' (CA1 20211_1_0028)

(20) taim te wong pa namwi a tamong imat tu niong

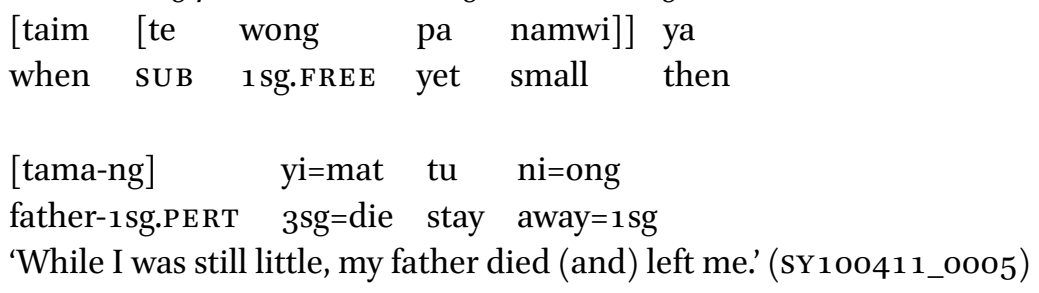

There are several 'native' Paluai forms that can be used instead of taim, such as no, pwotnan and pêng, and some speakers make a quite conscious effort to stick to these. It is possible, however, that the use of this array of terms as temporal subordinate clause markers only came about as a result of contact with TP, and a consecutive need arising in bilingual speakers to have a Paluai alternative. Many Oceanic languages do not have an overt form to mark subordination, but use simple juxtaposition, with the dependency relation having to be inferred from the context (Lynch, Ross and Crowley, 2002: 53). It may be the case that bilingual speakers tried replicating patterns in their one language to 
express temporal dependencies in the other, which was why taim came to be used, and 'native' forms were invented as a consequence.

\section{Convergence of Semantic Categories}

Another result of the ongoing pressure of $\mathrm{TP}$, which is less perceptible in some cases, is the loss of semantic categories in Paluai due to a many-to-one correspondence of categories in Paluai and TP, respectively. In this context the work of Malcolm Ross (e.g. Ross, 1996, 2001, 2007) on 'metatypy' is relevant. Ross discusses contact-induced change in Takia, an Oceanic language spoken on Karkar Island off the north coast of New Guinea, that is in close and extended contact with Waskia, a Papuan language. In Takia, not only borrowing of individual lexical and grammatical items took place, but a reorganisation of the entire semantic and grammatical systems. For instance, in Takia there are many calques of lexical collocations, the adpositional system shows the same range of meanings as that of Waskia, and the language switched from svo to sov constituent order. This, according to Ross (2007), is metatypy proper, which should be distinguished from both lexical and grammatical borrowing and from calquing, because it affects the language system as a whole. As one of the reasons why metatypy occurs he suggests that bi- or multilingual speakers are pressed to reduce the cognitive load that follows from the need of expressing the same thoughts in two or more languages. Semantic and grammatical restructuring, leading to greater intertranslatability, would have this effect.

Since Paluai and TP are structurally very similar, there may be a reduced need for grammatical restructuring of the former, under pressure from the latter. We do, however, find a restructuring of semantic categories. In many cases, there is a many-to-one correspondence between Paluai and TP in terms of semantic distinctions. A number of these instances are discussed below.

\subsection{Numerals and Numeral Classifiers}

In this domain, we find both lexical borrowing and semantic attrition. Paluai numerals, especially for the higher numbers, are complex, long forms, and as a result they have been almost completely replaced with English numerals. Most people will still be able to count from one to ten, but not much higher than that. In addition, numerals receive a suffix based on the semantic class of the noun. The only semantic distinction that is still consistent in present-day Paluai is the one between animate beings and inanimate objects. Other noun classes are now only represented for the numeral one (and sometimes two and three), which usually has a suppletive form. There used to be separate noun classes for elongated things such as trees and canoes, flat two-dimensional things, 


\begin{tabular}{ll}
\hline saya & place, area of land, village \\
sakam & leaf \\
sei & elongated object \\
seleuk & part of elongated object \\
sikil & leaf, wing, betel pepper fruit \\
sip & inanimate object (residue or default category) \\
som & animate being \\
sum(ut) & flat object, grass skirt \\
supu & heap, bundle, group (of e.g. fruit or people) \\
sui & heap of fruits \\
sek & half of elongated object \\
sopwol & half of round object \\
\end{tabular}

leaves, places and perhaps many more. The substitution of Paluai numerals with English numerals has led to the almost complete disappearance of the classifier system, because the English numerals do of course not make this semantic distinction. Since there are fewer semantic categories than there used to be, this is an example of semantic attrition.

\subsection{Grammatical Number}

Paluai makes a four-way distinction in the grammatical number system, shown on pronominal forms: singular, dual, paucal (referring to a few people or entities) and plural (referring to more than a few people or entities). тр has a three- or four-way number distinction between singular, dual, sometimes trial, and plural. Anecdotal evidence suggests that the Paluai paucal number may be on its way out, perhaps under influence from TP. There are two trends, it appears: either to use the paucal to refer to three people or entities exclusively, or to not use the paucal at all and exclusively use the plural to refer to quantities greater than two. This phenomenon appears to be particularly common in younger speakers. It is an interesting development, because the paucal category in Paluai and other Oceanic languages may in fact have developed out of a trial (Rene van den Berg, p.c.). It may still be a rather unstable category in the grammar, showing cyclical changes of going in and out of use.

\subsection{Sibling Terms}

The Paluai kinship system is rather complex, and in order to use the right kinship term a speaker has to have detailed knowledge of descent lines. Not only 'real' siblings, but also parent's sibling's children are classified as one's own 


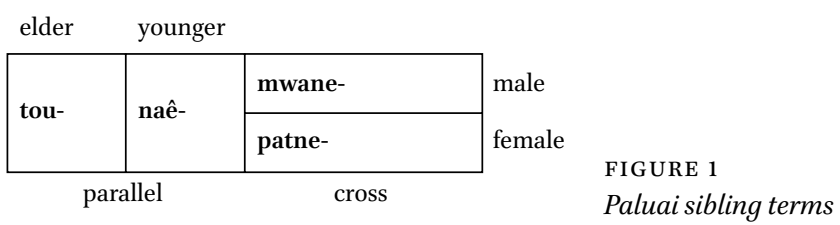

siblings. Distinction, which is schematically represented in Figure 1, is made based on speaker's own sex (represented in the lines), relative sex (whether the same or different from that of the speaker; first and second broad column), and relative age (the two narrow columns that divide up the first broad column). A male speaker refers to a cross-sex (i.e. female) sibling with a different term than the one a female speaker uses to refer to a male sibling. For same-sex siblings, male and female speakers use the same terms, but then relative age matters: there is a separate term for an older and a younger sibling. In order to use the right term with a classificatory sibling, a speaker furthermore has to know whether their father or mother is senior to their own father or mother.

Most likely partly because of the complexity of the system, the use of Paluai sibling terms is on the wane, and terms are being replaced with TP brata or sista. Interestingly, brata used to refer to a sibling of the same sex, for both male and female speakers, and sista referred to a sibling of the opposite sex. Due to increasing influence of English, the terms are now almost exclusively used, both in TP and when borrowed into Paluai, with their English meanings. Kinship terminology is another domain where semantic attrition has occurred: category distinctions that were made by the Paluai terms, such as older vs. younger sibling, are no longer made because the English and/or TP terms do not specify for this.

\subsection{Physical Property Terms}

One last domain where there is a many-to-one correspondence is that of physical property terms. Most physical properties are expressed by adjectives or stative verbs in Paluai. Often each of the terms is very precise, and used to refer to a property of one specific type of material or object. In TP, there is usually only one "umbrella" term related to this semantic subfield, which is used for a range of objects and meanings. An example is the group of Paluai adjectives related to the property STRONG. At least four are attested:

- paiwon 'hard, tough' (derived from the stative verb paoy 'to harden')

- sasawan 'strong, of smell'

- parayoyop 'strong, powerful'

- ngeingein '(very) strong, of sun' 
In TP there is only one term, strong, to represent this variety. It seems that in Paluai, the diversity of physical property terms is depleting due to the influence of the cover-all TP term. Nowadays, phrases such as sin paiwon 'strong sun' (lit. 'hard sun') or wong paiwon 'I am strong' (lit. 'I am hard') are frequently heard.

It seems that only one Paluai term, paiwon, is taking the place of the range of terms that used to be there. It is an interesting question why it would be this term, out of the range, that gets selected. Paiwon could be the most general Paluai term, and it is certainly possible that there are genre effects at work here: some physical property terms could be restricted to or preferred for certain genres, e.g. narratives, or belong to a more elaborate or poetic register, and not be frequently used in day-to-day discourse. It can nevertheless be argued that semantic attrition is in progress in Paluai. Not only do many, in particular younger, speakers not use certain terms, but they also do not seem to have an active or even passive knowledge of them any longer and thus have no choice between registers: many younger speakers were for instance not familiar with the term sasawan, when prompted. Of course, one could say that this is a regular feature of language change, and that many younger speakers of languages like Dutch or English also have a vocabulary that is different from older generations. Still, their vocabularies would not be significantly different in size or specification from those of the older generations, which does seem to be the case with the shift from Paluai to TP semantic categories.

A similar process of semantic attrition may be at the root of the situation with the TP loan wasim 'wash' as mentioned earlier in this paper. In Paluai, the verb forms related to the act of 'washing' differ slightly depending on whether they describe a transitive event or not and if transitive, whether the object is human or not. Thus, there are the intransitive form nu 'bathe', and the transitive forms nup 'bathe, wash a person' and nul 'wash an object (e.g. food, dishes, laundry). ${ }^{5}$ When a speaker is unsure about which verb to use, he or she may revert to TP wasim (or waswas, in case of an intransitive event) to avoid using the 'wrong' verb. When the Paluai forms are used less frequently, it means in turn that speakers' intuitions about them will become weaker. This may cause a vicious circle leading to the fallout of Paluai forms.

\section{Conclusions}

Contact-induced language change is a multi-faceted phenomenon. Quite often, a principled reason for why a certain form is adopted into the Paluai

5 The formal relationship between these verbs remains opaque: $-p$ and $-l$ are not attested as transitivising suffixes anywhere in the grammar. 
system appears at least plausible. igat constructions are used because they are more fortunate from a pragmatic viewpoint, inap may be borrowed because it does not carry additional apprehensive semantics, and taim specifies relations which may otherwise have been left implicit. In other cases, TP forms are on the increase because speakers appear to have lost intuitions as to the exact semantics of the Paluai terms. Thus, it appears that contact-induced change in Paluai is at least partly usage-driven. Since the two languages are structurally so similar, and probably always have been, the amount of grammatical restructuring is expected to be lower than may have been the case with two typologically much more diverse languages in a similar contact situation. Without historical records for Paluai, it is hard to say, however, how much convergence between the two languages has taken place already. Nevertheless, the effect of lexical calquing on Paluai semantic categories is probably significant.

Discussion and Suggestions for Further Research

What do these developments mean for the future of Paluai and other vernaculars of $P N G$ ? First of all, if we consider semantic category restructuring a type of language loss or language shift, it has to be remarked that within the speech community it appears to stay under the radar when compared to other types of language loss or shift. Paluai speakers pride themselves on the fact that their children still acquire the local vernacular as their first language, in contrast to the situation in other speech communities. It is said that in Mouk village, the children of the Titan speakers no longer acquire this language at all. But the language that Paluai children learn nowadays, although they do acquire it, is probably a quite different, and some would say "depleted", variety compared to what was spoken a few decades ago.

To add to this, like every language, TP is not in stasis either. Several authors (e.g. Romaine, 1992b, 2000; Verhaar, 1995) have argued that TP is in a process of decreolization due to increased contact with its lexifier language English, in particular in urban areas. One could therefore say there is an urban variety of the language as opposed to a rural one (or more likely, rural ones), which are not always mutually intelligible. As English is becoming ever more present also within rural communities, through the increasing use of the internet and social media, it is likely that decreolization is on the rise in rural varieties as well. There may also be an increased likelihood of direct borrowing from English into the vernaculars. As pointed out, with many borrowed items it is hard to say which road they followed: directly from English or 'via' TP. There is, however, at least one function word in Paluai that seems to have been adopted 
straight from English: so used as a conjunction or discourse marker in an evaluative sense. TP does not at all have a form with a similar function, either using English or substrate lexical material; thus it must be concluded that this form was borrowed straight from English.

A conceivable outcome of the process of change is that vernaculars converge, via TP, and thus become gradually more similar to each other. The Admiralties languages (which number about 32 in total) have not been described in great detail, but it is possible that each has or had its own specific ways to mark temporal subordinate clauses, or to mark deontic modality, and so on, and that in each of these languages the original construction has been or will be replaced by the same TP form. Eventually, this will decrease linguistic diversity. On the other hand, there always has been and always will be language contact, in particular in such a highly multilingual area as PNG, and thus also contact-induced language variation. Since each language is different, contact phenomena are also likely to be slightly different in each language. In this sense, contact may even lead to more language diversity.

As is evident from work such as e.g. Trudgill (2011), language contact can lead to either simplification or complexification of the languages or varieties in question, or perhaps even both simultaneously. Very roughly, a distinction can be made between two types of situations in which a language is acquired: stable, uninterrupted intergenerational transmission from adults to children which often leads to grammatical complexification, or a high contact situation in which a language is learned by large numbers of adults, which often leads to simplification. ${ }^{6}$ When, in the first type of situation, there is a stable tradition of bi- or multilingualism, where children acquire two or more varieties simultaneously over many generations, often we see complexification of one or more of the varieties involved due to additive borrowing, in which 'new features derived from neighbouring languages do not replace existing features but are acquired in addition to them' (Trudgill, 2011: 27). This seems to be what we are seeing in the case of Paluai and TP. Already for several generations, children have acquired Paluai and TP simultaneously, and on a synchronic level TP lexical items and constructions are added to the repertoire of speakers. This is done, synchronically at least, not fully at the expense of Paluai items and constructions, making the language as it currently is more complex. Only time can tell whether this situation will stabilize, and speakers will continue to have several options, or whether the Paluai items and constructions will be ousted.

6 In actual fact, many more factors play a role in linguistic variation and change, as Trudgill also discusses in great detail. Also, phenomena can probably be modelled more accurately if the differences in situations are viewed as a continuum rather than as discreet categories. 
Thus, it is very important to distinguish synchrony from diachrony when this kind of phenomenon is discussed.

What is also fascinating is whether a creole language such as TP would be more 'suitable' for borrowing, or is more likely to be borrowed, due to its higher transparency. After all, pidgin languages, which are seen as the sources out of which creole languages develop, originate in a type of situation which sits at one extreme end of the continuum described above: a large group of adult learners from different linguistic backgrounds, who do not know each other and do not share a language in common, but who need to have a means of communication nonetheless. As a result of this, pidgins show enormous simplification, with often greatly reduced phoneme inventories and paradigms and no morphology, and are thus very transparent; this enables an adult learner population to get by with them with relative ease. This transparency is often carried over when a pidgin develops into a creole, which continues to show little inflectional morphology, instead expressing most categories periphrastically. More comparative research may show that bilingual speakers prefer items with greater transparency to transfer between their languages, which would indicate that creoles are more likely to get borrowed from than other languages with more inflection.

In any case, language contact situations are incredibly dynamic. As mentioned, there is a variety of literature about the substrate influence of local vernaculars on the creoles in the area. This paper suggests that at the same time, the creoles are most likely also exercising their influence over the local vernaculars in various ways. It is important to acknowledge this two-way process when undertaking linguistic and sociolinguistic studies that focus on creolization. Moreover, there probably is variation within the speech communities as well, as not all speakers or groups of speakers will show the same behaviour in their use of multiple codes. Age is the most obvious diversifying factor in this regard: each cohort of speakers will have different input and thus different exposure to a 'mix' of languages. As mentioned above, adolescents may be important drivers of language change, and it may be to this group that TP has highest appeal. Also, speakers are not stable in their use of multiple codes throughout their lives. Cases have been reported (Miriam Meyerhoff, p.c.) of Vanuatu children using only Bislama, and twenty years later, as adults, speaking the local vernacular fluently and complaining that their own children refused to use anything other than the creole! Other social variables, such as sex, clan membership and individual language history, may play a role too. More variationist sociolinguistic research in small speech communities is needed, in order to spread more light on the processes behind contact-induced change and language variation here. 


\section{Acknowledgements}

This paper was presented, in various forms, at several conferences and departmental meetings. I wish to thank all participants on these occasions for their valuable input, which helped me enormously to formulate, solidify and expand my ideas. Thanks are also due to an anonymous reviewer for helpful suggestions. In particular, I wish to acknowledge my debt to the speakers of Paluai, on Baluan Island, for their friendship, support and hospitality during my field stays, and for sharing their beautiful place, language and culture with me.

\section{References}

Aitchison, Jean. 2003. Psycholinguistic perspectives on language change. In The Handbook of Historical Linguistics, Brian D. Joseph and Richard D. Janda (eds.), 736-743. Oxford: Blackwell.

Crowley, Terry. 1990. From Beach-la-Mar to Bislama: The emergence of a national language. Oxford: Oxford University Press.

Crowley, Terry. 2000. "Predicate marking" in Bislama. In Processes of language contact: studies from Australia and the South Pacific, Jeff Siegel (ed.), 47-74. Montreal: Fides.

Dixon, Robert M.W. 2012. Basic linguistic theory. Volume 3: further grammatical topics. Oxford: Oxford University Press.

Jenkins, Rebecca Sue. 2005. Language Contact and Composite Structures in New Ireland. Dallas, Texas: SIL International.

Keesing, Roger M. 1988. Melanesian Pidgin and the oceanic substrate. Stanford, CA: Stanford University Press.

Keesing, Roger M. 1991. Substrates, Calquing and Grammaticalization in Melanesian Pidgin. In Approaches to grammaticalization, Elisabeth Closs Traugott and Bernd Heine (eds.). Amsterdam: John Benjamins.

Kerswill, Paul. 1996. Children, adolescents, and language change. Language variation and change 8: 177-202.

Kerswill, Paul and Ann Williams. 2005. New towns and koineization:linguistic and social correlates. Linguistics 43: 1023-1048.

Lambrecht, Knud. 1994. Information structure and sentence form: topic, focus, and the mental representations of discourse referents. Cambridge: Cambridge University Press.

Lynch, John, Malcolm Ross and Terry Crowley. 2002. The Oceanic Languages. Richmond, Surry: Curzon.

Meyerhoff, Miriam. 200o. The emergence of creole subject-verb agreement and the licensing of null subjects. Language Variation and Change 12(2): 203-230. 
Meyerhoff, Miriam. 2001. Another look at the typology of serial verb constructions: The grammaticalization of temporal relations in Bislama (Vanuatu). Oceanic Linguistics 40: 247-268.

Meyerhoff, Miriam. 2009. Replication, transfer, and calquing: Using variation as a tool in the study of language contact. Language Variation and Change 21: 297-317.

Mosel, Ulrike. 1980. Tolai and Tok Pisin: the influence of the substratum on the development of New Guinea Pidgin. Canberra: Pacific Linguistics.

Nettle, Daniel. 1999. Linguistic diversity. Oxford: Oxford University Press.

Romaine, Suzanne. 1992a. The Inclusive/Exclusive Distinction in Tok Pisin. Language and Linguistics in Melanesia: Journal of the Linguistic Society of Papua New Guinea 23(1): 1-11.

Romaine, Suzanne. 1992b. Language, Education and Development: Urban and Rural Tok Pisin in Papua New Guinea. Oxford: Clarendon Press.

Romaine, Suzanne. 1995. The Grammaticalization of Irrealis in Tok Pisin. In Modality in Grammar and Discourse, Joan L. Bybee and Suzanne Fleischman (eds.), 389-427. Amsterdam: John Benjamins.

Romaine, Suzanne. 2000. Language in Society: an Introduction to Sociolinguistics. Oxford: Oxford University Press.

Ross, Malcolm D. 1985. Current use and expansion of Tok Pisin: effects of Tok Pisin on some vernacular languages. In Handbook of Tok Pisin (New Guinea Pidgin), Stephen A. Wurm and Peter Mühlhäusler (eds.), 539-556. Canberra: Pacific Linguistics.

Ross, Malcolm D. 1996. Contact-induced change and the comparative method: Cases from Papua New Guinea. In The comparative method reviewed: Regularity and irregularity in language change, edited by Mark Durie and Malcolm Ross, 180-217. New York: Oxford University Press.

Ross, Malcolm D. 2001. Contact-induced change in Oceanic languages in North-West Melanesia. In Areal diffusion and genetic inheritance, Alexandra Aikhenvald and Robert M.W. Dixon (eds.), 134-166. Oxford: Oxford University Press.

Ross, Malcolm D. 2007. Calquing and metatypy. Journal of Language Contact Vol. THEMA 1: 116-143.

Ross, Malcolm D. 2013. Diagnosing contact processes from their outcomes: The importance of life stages. Journal of language contact $6: 5^{-47}$.

Sankoff, Gillian. 1994. An historical and evolutionary approach to variation in the Tok Pisin verb phrase. Parasession on Variation and Linguistic Theory: Chicago Linguistic Society 30: 293-320.

Schokkin, Dineke. 2014. A grammar of Paluai, the language of Baluan Island, Papua New Guinea. Doctoral dissertation, James Cook University.

Siegel, Jeff. 1998. Substrate Reinforcement and Dialectal Differences in Melanesian Pidgin. Journal of Sociolinguistics 2(3): 347-373. 
Smith, Geoff P. 2002. Growing up with Tok Pisin: contact, creolization, and change in Papua New Guinea's national language. London: Battlebridge.

Thomason, Sarah G. and Terrence Kaufman. 1988. Language Contact, Creolization, and Genetic Linguistics. Berkeley, CA: University of California Press.

Timberlake, Alan. 2007. Aspect, tense, mood. In Language typology and syntactic description, edited by Timothy Shopen, 280-333. Cambridge: Cambridge University Press.

Trudgill, Peter. 2011. Sociolinguistic typology: Social determinants of linguistic complexity. Oxford: Oxford University Press.

Verhaar, John. 1995. Towards a reference grammar of Tok Pisin: An Experiment in Corpus Linguistics. Honolulu: University of Hawai'i Press. 